\title{
Application of the Extended Finite Element Method to the Penetration Process for Concrete Target
}

\author{
WANG Feng ${ }^{1, a}$, YU Jing ${ }^{1, b}$, TANG Jian-cheng ${ }^{2, ~ c}$, YU Kai-ping ${ }^{1, d}$, WANG \\ Nai-long ${ }^{1, \mathrm{e}}$ \\ ${ }^{1}$ Army Officer Academy of PLA, Hefei, 230031, Anhui, China \\ ${ }^{2}$ Xuzhou Technology Normal College, Xuzhou, 221151, Jiangsu, China \\ awangfeng0706@163.com, byujing0830@163.com, ctjc505@163.com, \\ dyukson@163.com, ${ }^{\mathrm{e}}$ chromle@hotmail.com
}

\begin{abstract}
Keywords: extended finite element method (XFEM); discontinuous problem; crack growth;
\end{abstract} numerical simulation; penetration

\begin{abstract}
The XFEM makes research within a standard finite element framework and needs not mesh repartition to geometric and physical interface, so it reserves all merits of the conventional finite element method and it is the most effective numerical method to solve discontinuous dynamic problems so far. The XFEM was applied to the penetration process for concrete target in the paper, and the displacement mode of elements with cracks and fracture criterion were presented. Then the weak solutions of control equations were discretized in different areas. The numerical examples for steel rod penetrating in the concrete target concluded that the method and program were reasonable and effective. The effect discipline of crack growth to the concrete material penetration process was summarized, and it would establish theoretic base for the further application of the XFEM.
\end{abstract}

\section{Introduction}

In the process of numerical calculation of transient dynamics, when solving the distortion problem which includes high speed penetration, crack dynamic growth, mental sharp distortion and so on, mesh would be warped severity and bring on the discontinuousness and distortion which is unequal to the initial mesh. The conventional finite element method (CFEM) often uses mesh repartition to solve the discontinuous problem, thus the computational efficiency is very low and even incapable. The extended finite element method (XFEM) was used normally in 2000[1], which was developed for the discontinuous problems. The idea of the XFEM can solve the difficulty perfectly caused by the discontinuous problem when using CFEM. The XFEM makes research within a standard finite element framework and needs not mesh repartition for geometric and physical interface, so it reserves all merits of the CFEM. It is the most effective numerical method for solving discontinuous problem so far.

The XFEM developed very rapidly in last decennary. According to the partition of unity, Sukumar et al [2] added discontinuous function and two-dimensional crack tip approximately field to CFEM for solving the three-dimensional crack problem based on the XFEM. Some numerical problem relating to the XFEM were discussed by Tian-tang YU [3], such as interface definition using level set method, strength node and pattern and the choice of enhanced area.

\section{Displacement mode of the XFEM}

In the process of generating the CFEM meshes, the existence of some defects can't be ignored, such as crack, holes and inclusions. That is to say, the sides of the elements must be consistent with the geometry boundaries, so the mesh repartitions are very complex. However, such difficulties can be solved perfectly by using the XFEM, in which the finite element meshes need not keep coordination with the inner boundary. The XFEM modeling can be divided into two parts. Firstly the entire region can be meshed without considering the inner boundary. Secondly additional 
functions related to the inner boundary will be added to the unit shape function, for improving the finite element approximation space. The general form of the XFEM displacement mode is shown as

$$
u(x)=\sum_{i \in I} N_{i}(x)\left(u_{i}+a_{i} \phi_{i}(x)\right) .
$$

where $I$ is the set of all nodes, $N_{i}$ is the shape function of node $i$ in CFEM, $u_{i}$ is the continuous section of displacement vector on node $i, a_{i}$ is the additional freedom of node $i, \phi_{i}(x)$ is the additional discontinuous function considering the influence of discontinuous structure.

\section{Infirm solution form of control equation and region scatteration}

The balance equation and boundary condition of discontinuous medium mechanics problem involving with cracks are as follows.

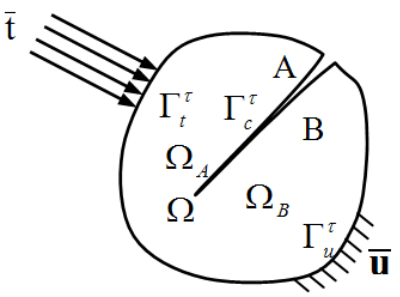

$$
\left\{\begin{array}{lll}
\nabla \cdot \boldsymbol{\sigma}+\mathbf{b}=0 & \text { in } \Omega & \\
\mathbf{u}=\overline{\mathbf{u}} & \text { on } \Gamma_{u}^{\tau} & \\
\boldsymbol{\sigma} \cdot \mathbf{n}=\overline{\mathbf{t}} & \text { on } \Gamma_{t}^{\tau} & \\
\boldsymbol{\sigma} \cdot \mathbf{n}=0 & \text { on } \Gamma_{c}^{\tau} \quad(\text { crack open) }) \\
\boldsymbol{\sigma}^{A} \cdot \mathbf{n}^{A}+\boldsymbol{\sigma}^{B} \cdot \mathbf{n}^{B}=0 & \text { on } \Gamma_{c}^{\tau} \quad(\text { crack close) } \\
{\left[\mathbf{u}_{\mathbf{N}}\right]=\mathbf{u}^{A} \cdot \mathbf{n}^{A}+\mathbf{u}^{B} \cdot \mathbf{n}^{B} \leq 0} & \text { on } \Gamma_{c}^{\tau} \quad(\text { contact condition) }
\end{array} .\right.
$$

Fig.1. Sketch map of all sorts of boundary at time $\tau$

Where $\mathbf{b}$ is the unit volume force, $\boldsymbol{\sigma}$ is the stress tensor, $\mathbf{n}$ is the outer normal vector, $\mathbf{u}$ is the displacement vector, $\overline{\mathbf{t}}$ is the surface force on boundary, $\nabla$ is the Hamilton arithmetic operators, $\Omega$ is the set not including cracks, $\Gamma_{u}^{\tau}$ is the displacement boundary of time $\tau, \Gamma_{t}^{\tau}$ is the force boundary of time $\tau, \Gamma_{c}^{\tau}$ is all crack surface, $\mathbf{n}^{A}$ and $\mathbf{n}^{B}$ are the outer normal vectors of corresponding free crack surface (Fig.1).

Considering geometry and constitutive equation, the infirm solution form is

$$
\left\{\begin{array}{l}
\mathbf{K}_{u u} u+\mathbf{K}_{u a} a-f^{\text {ext }}=0 \\
\mathbf{K}_{u a}^{T} u+\mathbf{K}_{a a} a-q^{e x t}+\mathbf{G}^{T} \lambda=0 \\
\mathbf{G} a=0
\end{array} \quad \text { or } \quad\left[\begin{array}{ccc}
\mathbf{K}_{u u} & \mathbf{K}_{u a} & 0 \\
\mathbf{K}_{u a}^{T} & \mathbf{K}_{a a} & \mathbf{G}^{T} \\
0 & \mathbf{G} & 0
\end{array}\right]\left[\begin{array}{l}
u \\
a \\
\lambda
\end{array}\right]=\left[\begin{array}{c}
f^{\text {ext }} \\
q^{\text {ext }} \\
0
\end{array}\right] .\right.
$$

Where $\mathrm{K}_{u u}$ is the structure stiffness matrixs of CFEM, $\mathrm{K}_{a a}$ is the stiffness matrixs including appendant freedom, $\mathrm{K}_{u a}$ is the stiffness matrixs coupling $u$ and $a, a$ is the appendant freedom, $\lambda$ is the Lagrange multiplier, $f^{\text {ext }}$ is the external load, $q^{\text {ext }}$ is the cohesive force.

\section{Fracture criterion}

The common fracture criterions of the compound crack have three kinds, the biggest shear stress, minimum strain density factor, and energy release rate. The above-mentioned criterions are generally concerned the calculation of stress intensity factor. We assume that if the element occur crack, the crack will penetrate the whole element. The average stress is treated as fracture criterion.

(1) When main stress of average stress exceeds tensile intensity of material, the element will crack.

(2) The tendency of crack growth verticals main stress direction.

To simplify calculation, crack growth should comply following assumption.

(1) Crack will grow along element side (or diagonal), in other words, the crack always directly extends from one side (or diagonal) to another side in element (or diagonal).

(2) Considering compound crack growth causing intersecting and coinciding of many cracks in penetration calculation, the slide surface calculating will become extremely complicated. If having a crack, we will only consider present crack growth but not the new crack in element.

\section{Numerical examples}

A series of experiments about steel ogive-nosed rod penetrating in the concrete target were carried out by Hanchak, and many valuable experiment data were obtained. We made the XFEM 
program with above numerical method and simulated those penetration processes according to the experiment results. In order to research the basic discipline of crack growth in high speed penetration, the microcrack was added to the target. The crack of direction and length were presented in the crack, but the numerical value of expanding displacement was zero. The initially meshes distributing figure is shown in Fig.2.

The model of steel rod adopts fluid elastoplasticity model and relevant parameter of physical and calculation $[4,5]$. The model concrete target involves two kinds, and the relative model containing damage rate is used to elements without cracks, and the cohesive crack model is used to elements with cracks. The cohesive crack model is based on suppositional crack model, and it essential idea is as follows.

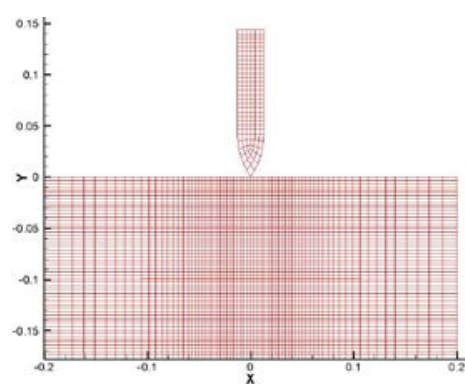

Fig.2. The initially meshes distributing figure

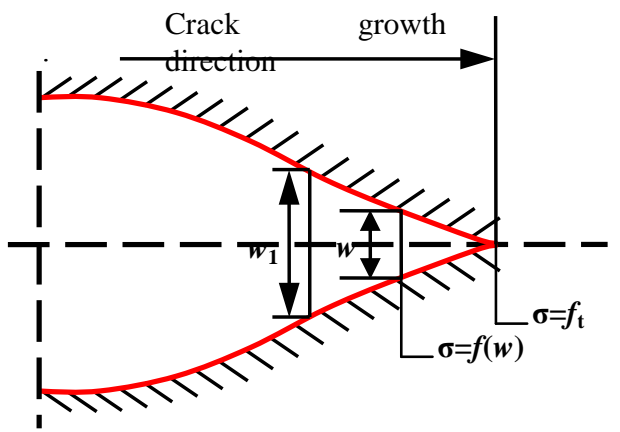

Fig.3. The stress distributing of crack surface

(1) When the crack tip stress comes up to the intensity limit , the crack will begin to grow.

(2) When the crack expands, the cohesive stress $\sigma$ of crack surface will decrease gradually along with the increase of crack expanded degree $w$. It is shown in Fig.3. The work will be done by cohesive stress in the process of crack expanding, so some energy will be absorbed.

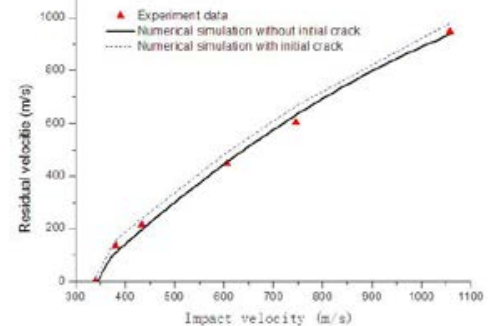

Fig.4. Comparison of residual velocities

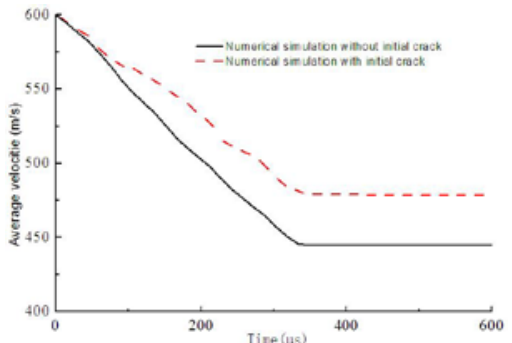

Fig.5. Average velocity-time curve

Normal penetration with different initial velocities were simulated. Comparison of residual velocities between numerical simulation and experiment of penetration is shown in Fig.4. It is easily seen that the numerical simulation without initial crack are in good agreement wit the experimental result for both normal and oblique penetrations. The result indicates that the existence of the initial crack leads to the reduction of the protection ability and enhancing the residual velocity.

The average velocity-time curve of penetration with initial velocity $600 \mathrm{~m} / \mathrm{s}$ is shown in Fig.5. The figure shows that the average velocity is approximately linear reducing in penetration period. It indicates that the negative acceleration and the penetration resistance approach a constant. Once the target is perforated, the velocity retains constant and the numerical value of penetration resistance is down to zero. Meanwhile, the higher average velocity of penetrating in the target with initial crack illustrates that the existence of the initial crack leads to the decrease t of the protection ability.

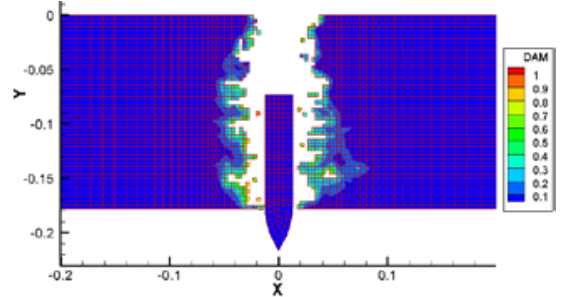

(a) Without initial crack

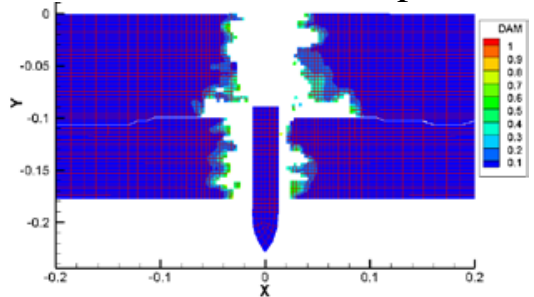

(b) With initial crack

Fig. 6 Damage contours of normal penetration with initial velocity $6000 \mathrm{~m} / \mathrm{s}$ at $\mathrm{t}=440 \mu \mathrm{s}$ 
The damage contours of normal penetration with the same initial velocities and time are shown in Fig.6. From this figure, we can see that the damage of approaching rod is more obvious in the penetration with initial crack. In Fig.6.(b), with the tension effect caused by rod penetration, the crack extends gradually to the sides and ends at the target boundary, but it has no obvious expanding in the extending process. It is because of the mechanical property of concrete material. The concrete is brittle material, and its tensile strength is lower greatly than the compression strength. The elastic and plastic strains of elements are very small with the stress effect. When the element stress reaches material tensile strength, the crack begins to extend. With more element stress, the cumulative damage causes the element to destroy, but the crack expand displacement is not evident, and it is obvious different from mental material.

\section{Summary}

The thought of the XFEM is simple, but it can easily resolve any discontinuous problem. The XFEM is especially suitable for solving the dynamic cracks growth and the program design only need to make the appropriate changes in the CFEM program. The XFEM is a new numerical method with the broad prospects for the development, and it can provide an effective platform for complex discontinuous numerical analysis and computation.

\section{Acknowledgements}

This work was financially supported by the Scientific Research Foundation of Army Officer Academy (2014XYJJ-086).

\section{References}

[1] Daux C, Moes N, Dolbow J, et al. Arbitrary branched and intersecting cracks with the extended finite element method [J]. International Journal for Numerical Methods in Engineering, 2000, 48: 1741-1760.

[2] Sukumar N, Moes N, Moran B, et al. Extended finite element method for three-dimensional crack modeling [J]. International Journal for Numerical Methods in Engineering, 2000, 48:1549-1570.

[3] Tian-tang YU. Numerical aspects of the extended finite element method [J]. Rock and Soil Mechanics, 2007, 28: 305-310. (in Chinese)

[4] Feng WANG, Xiao-jun WANG, Xiu-zhang HU, et al. Oblique penetration of an ogive-nosed rod into the aluminum target. Explosion and Shock Waves, 2005, 25(3): 265-270. (In Chinese)

[5] Feng WANG, Xiu-zhang HU, Xiao-jun WANG, et al. Numerical Simulations of an Ogive-noded Rod Penetration in the Concrete Target by Finite Element Method J]. Journal of Ballistics, 2008, 20(2): 1-4(In Chinese) 